\title{
Latent Acquisition of Timed Responses in Cerebellar Cortex
}

\author{
Tatsuya Ohyama and Michael D. Mauk \\ Department of Neurobiology and Anatomy, University of Texas Medical School, Houston, Texas 77225
}

\begin{abstract}
Evidence indicates that rabbit eyelid conditioning is mediated by plasticity in the interpositus cerebellar nucleus and in cerebellar cortex. Although the relative contributions of these sites are not fully characterized, evidence suggests that plasticity in the cerebellar cortex influences conditioned response amplitude and timing, whereas plasticity in the interpositus nucleus is necessary or permissive for conditioned response expression. Recent empirical and computational analyses suggest that, during training, plasticity is initially established in the cerebellar cortex, whereas conditioned response expression begins later as plasticity is induced in the interpositus nucleus. We used the dependence of response timing on the interstimulus interval (ISI) to test this latent learning hypothesis. Rabbits were initially trained using a tone conditioned stimulus (CS) with a relatively long ISI to a low-criterion threshold. The relative absence of plasticity in the interpositus nucleus was then examined via
\end{abstract}

A substantial body of evidence suggests that the plasticity necessary for rabbit eyelid conditioning occurs in both the anterior interpositus nucleus (AIN) and the cerebellar cortex (Raymond et al., 1996; Krupa and Thompson, 1997; Mauk, 1997). Plasticity in the cerebellar cortex appears to be required for the precise timing of the conditioned response, because permanent or reversible disconnection of the cerebellar cortex severely disrupts response timing (Perrett et al., 1993; Perrett and Mauk, 1995; Garcia and Mauk, 1998; Garcia et al., 1999; Medina et al., 2000). The residual conditioned responses spared after cerebellar cortex removal, with their short and relatively fixed latencies to onset irrespective of the training interstimulus interval (ISI), are thought to reflect either plasticity in the AIN at the mossy fiber to nucleus cell synapse (Garcia and Mauk, 1998) or excitability changes in nucleus cells (Aizenman and Linden, 2000). Evidence indicates that this cerebellar nucleus plasticity is necessary or permissive for the expression of the conditioned response (Clark et al., 1984, 1992; McCormick and Thompson, 1984; Yeo et al., 1985; Krupa and Thompson, 1997; Garcia et al., 1999). Consistent with this view, short-latency responses are revealed upon reversible disconnection of cerebellar cortex after but not before robust conditioning (J. F. Medina, K. S. Garcia, and M. D. Mauk, unpublished observations). A computer simulation of the cerebellum incorporating the two sites of plasticity further suggests that, early in training, plasticity is established first in the cerebellar cortex, whereas conditioned responses begin to appear later as plasticity in the AIN is established (Medina and Mauk, 1999).

We tested this latent learning hypothesis with a protocol that

\footnotetext{
Received Sept. 12, 2000; revised Oct. 24, 2000; accepted Oct. 25, 2000.

Correspondence should be addressed to Michael D. Mauk, Department of Neurobiology and Anatomy, University of Texas Medical School, Houston, TX 77225.
} E-mail:m.mauk@uth.tmc.edu.

Copyright (C) 2001 Society for Neuroscience 0270-6474/01/210682-09\$15.00/0 reversible disconnection of the cerebellar cortex. Later, to induce plasticity in the interpositus nucleus, subjects were trained to robust levels of conditioned response expression using a shorter ISI. Reversible disconnection of the cerebellar cortex at this time confirmed the presence of robust interpositus nucleus plasticity after the second phase. Subsequent probe trials with the long CS alone then revealed doublepeaked responses whose peaks were appropriately timed to the two ISIs. The results are consistent with the hypothesis that temporally specific learning occurs first in the cerebellar cortex before the appearance of conditioned responses. This latent learning is expressed only after plasticity is induced in the interpositus nucleus.

Key words: cerebellar cortex; eyelid conditioning; interpositus nucleus; latent learning; learning; plasticity; response timing

used (1) the dependence of conditioned response timing on the interstimulus interval [the ISI, or the interval between conditioned stimulus (CS) and unconditioned stimulus (US) onsets], and (2) the ability to query the status of learning-dependent plasticity in the AIN by reversible disconnection of the cerebellar cortex using infusions of the GABA antagonist picrotoxin into the AIN (Garcia and Mauk, 1998). In three experiments, rabbits were initially trained with a tone CS with a relatively long ISI until a low-criterion level of conditioned responding was attained or until a fixed number of trials had elapsed (phase I). We hypothesized that this training would establish latent learning in the cerebellar cortex with the absence of responding attributable to the absence of robust plasticity in the AIN. On the basis of evidence that the cerebellar cortex mediates conditioned response timing, we also anticipated that the latent learning would be temporally specific, that is, timed appropriately for the ISI. In phase II, each subject was trained for five sessions using the same CS but at a shorter ISI to induce plasticity in the AIN, which was confirmed using a second reversible disconnection of the cerebellar cortex. Based on the latent learning hypothesis, we expected that the latent learning initially established with the long ISI would be revealed in the form of double-peaked responses during subsequent tests with the long CS. The results supported this prediction.

\section{MATERIALS AND METHODS}

Subjects and surgery. Fifty-five naive New Zealand albino rabbits, each weighing $2.5-3.0 \mathrm{~kg}$, served as the subjects. The subjects were housed in individual cages, maintained on a fixed daily diet, and given water ad libitum. Treatment of animals and surgical procedures were in accordance with an approved animal welfare protocol.

Before training, animals were surgically prepared with a head stage, in some cases (experiment 1) with cannula implanted in the AIN. Subjects were first preanesthetized with $5 \mathrm{mg} / \mathrm{kg}$ acepromazine, and their skulls 


\section{A) NAIVE}

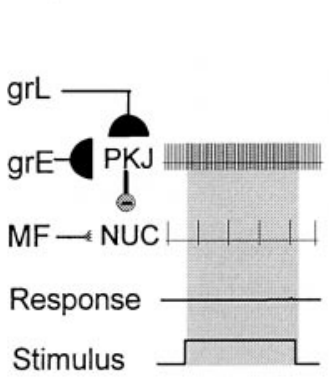

B) PHASE I

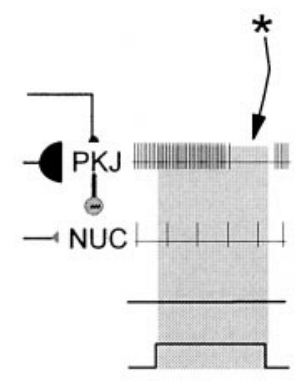

C) PHASE II

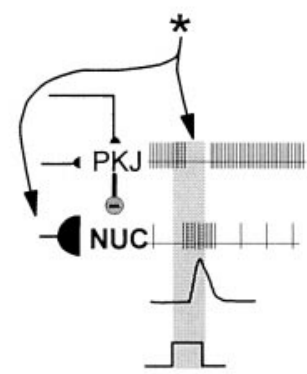

\section{D) LONG CS PROBES}
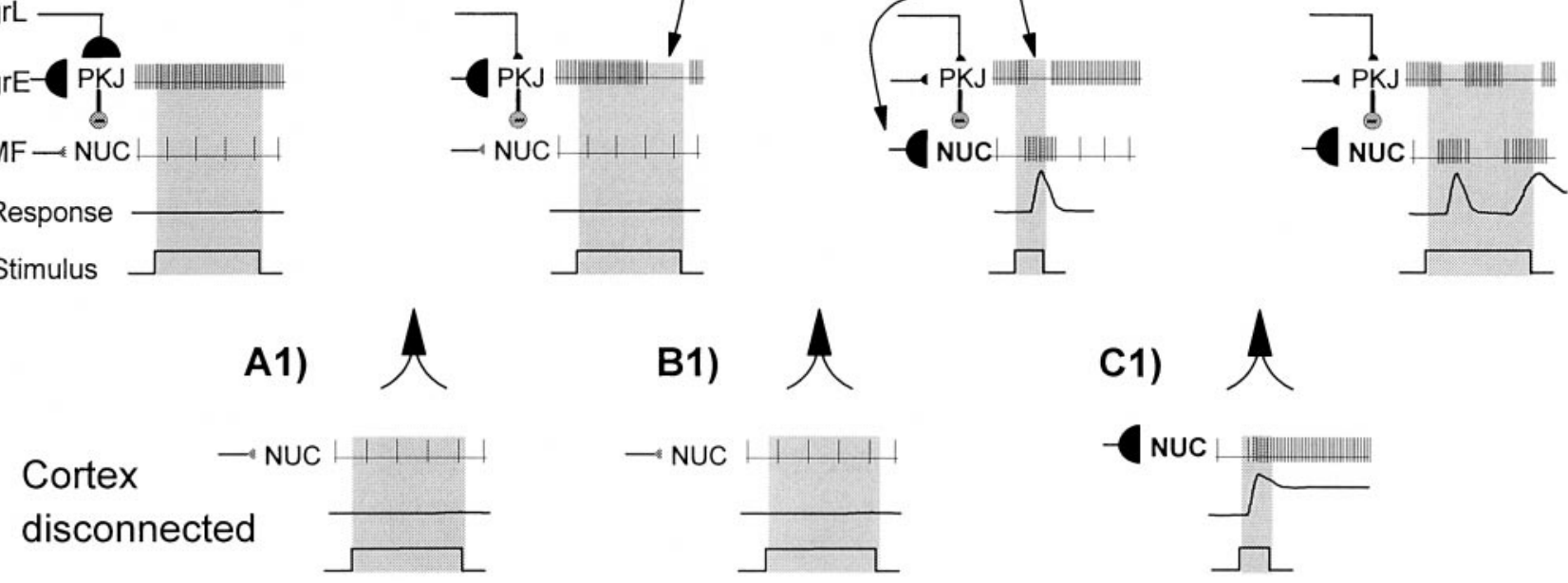

Figure 1. A schematic representation of the hypothesized latent learning in the cerebellum. The four top panels show the hypothesized state of the cerebellum at four stages of the experiment. The three bottom panels show the corresponding effects expected for reversible disconnection of the cerebellar cortex at the stage indicated by the arrows. $A$, In the naïve animal, Purkinje cells display robust ongoing activity that is not significantly altered by presentation of the CS. The combination of strong inhibition from Purkinje cells and the weak ability of the CS to activate cerebellar nucleus cells precludes the expression of a conditioned response. A1, Reversible disconnection of the cerebellar cortex at this stage has no effect because of the weak ability of CS-activated mossy fibers to affect nucleus cell activity. $B$, Early in training, we hypothesize that the Purkinje cells learn a well timed pause (asterisk), depicted as a weakening of granule cell $(g r)$ to Purkinje cell $(P K J)$ synapses that are active late in the CS ( $g r L)$, which could help elicit a conditioned response except for the absence of plasticity in the interpositus nucleus. Thus, the learning in the cortex is latent. B1, Reversible disconnection of the cerebellar cortex at this time should still have no effect attributable to lack of plasticity in the interpositus nucleus. $C$, Robust training with shorter presentations of the same CS induces a shorter-latency pause in Purkinje cell activity and plasticity in the interpositus nucleus (asterisks), depicted as a weakening of granule cell-Purkinje cell synapses active early in the CS ( $g r E)$ and a strengthening of the mossy fiber to nucleus synapses. The combination of these two forms of plasticity mediates the expression of a well timed learned response. Plasticity induced in phase I should not extinguish during this phase, to the extent that granule cell-Purkinje cell synapses active at the time of US occurrence differ in each phase. $C 1$, Because of the plasticity in the interpositus nucleus, reversible disconnection of the cerebellar cortex unmasks short-latency responses. $D$, We hypothesize that the latent learning can now be expressed with presentation of long CS probes, which should elicit double peaked responses. The first peak should correspond to the training in $C$, whereas the second peak is the latent learning corresponding to training in $B$.

were immobilized in a stereotaxic restrainer. Anesthesia was maintained with halothane (1-2\% mixed in oxygen), and sterile procedures were used throughout the operation. After exposing the skull, four holes were drilled to accommodate screws that served to keep the head bolt in place. For those subjects implanted with cannulas, a craniotomy was drilled lateral to lambda and covered with bone wax, and the head was positioned with lambda $1.5 \mathrm{~mm}$ ventral to bregma. A cannula with a 26 gauge stainless steel guide sheath and a 33 gauge internal cannula projecting 1.2 $\mathrm{mm}$ beyond the tip of the guide sheath was placed at stereotaxic coordinates corresponding to the left AIN $(0.7 \mathrm{~mm}$ anterior, $5.0 \mathrm{~mm}$ left lateral, and $14.0 \mathrm{~mm}$ ventral to lambda). The head bolt and cannula were then secured with dental acrylic, and any areas exposing the skull were sutured. Finally, two stainless steel stimulating electrodes were chronically implanted in the periorbital muscles rostral and caudal to the left eye. Subjects were allowed at least 1 week of recovery before experimentation.

Drugs and infusions. Experiment 1 involved infusion of the GABA antagonist picrotoxin (molecular weight of 602.6; Sigma, St. Louis, MO). A $2 \mathrm{~mm}$ solution was used for 12 subjects, and a $400 \mu \mathrm{M}$ solution was used for 11 other subjects. During any test session with the drug, $1 \mu \mathrm{l}$ of either solution was infused through the cannula at a rate of $0.5 \mu \mathrm{l} / \mathrm{min}$, using a $10 \mu \mathrm{l}$ syringe mounted on an electronic pump. The effective amount of picrotoxin infused on any test day was 2 nmol for 12 subjects, and 0.4 nmol for 11 other subjects. Subsequent testing was conducted at 45 or 30 min after the drug infusion for the 12 and 11 subjects, respectively.

Apparatus. Two identical custom-designed chambers were used in the experiments. The internal dimensions of each wooden chamber were $89 \times 64 \times 49 \mathrm{~cm}$ (width $\times$ length $\times$ height). A wooden divider $25 \mathrm{~cm}$ in height separated the chamber into left and right compartments, each accommodating a plastic restrainer in which the rabbits could be restrained. Each chamber was equipped with a speaker connected to an audio source module (Coulbourn Instruments, Allentown, PA) that generated tones and a pair of isolated pulse stimulators (model 2100;
Carlsborg, WA) that delivered electrical pulses through the implanted periorbital electrodes. The chambers were also equipped with two infrared emitter-detectors, each of which could be attached to the head bolt of an individual rabbit and directed at the left eye. The CS was a $1 \mathrm{kHz}$ pure tone $(85 \mathrm{~dB})$, and the US was a current pulse $(200 \mathrm{~Hz}, 1 \mathrm{msec}$ pulse width, $2.5 \mathrm{~mA}$ ). The duration of the CS varied according to the ISI and experiment. Stimulus presentation was controlled by custom-designed software operated on a computer situated adjacent to the two chambers.

Conditioning procedure. The number of subjects that served in experiments 1,2 , and 3 were 23, 24, and 8, respectively. At the start of each daily training or test session, immediately after placement in the chamber, the maximum amplitude of eyelid closure was calibrated by one of two methods. Twelve subjects in experiment 1 were presented with at least one (mean of $\sim 2$ ) pulse of an electric current $(2.5 \mathrm{~mA})$ through the periorbital electrode. All other subjects were touched in the vicinity of the left eye to elicit eyelid closure. Calibration was considered complete when the maximum amplitude of eyelid closure fell within a range of 5-7 $\mathrm{mm}$. During training or test sessions in which the CS and US were paired, the US always occurred $50 \mathrm{msec}$ before the termination of the CS. Each training session consisted of 12 nine-trial blocks (108 total trials), with each block including eight paired CS-US trials and one CS-alone trial. The mean intertrial interval was $30 \mathrm{sec}$ (range of 20-40 sec).

Data analysis. Data analysis was conducted using custom-designed software. Individual sweeps of eyelid movement (as detected by the amount of infrared light reflected from the eye) on each trial were obtained for a time period beginning $200 \mathrm{msec}$ before and continuing until $2300 \mathrm{msec}$ after CS onset. Measures of eyelid closure such as criterion onset latency (the time from CS onset at which amplitude of the response attained a criterion amplitude of $0.3 \mathrm{~mm}$ ), peak latency (the time from CS onset at which eyelid closure was maximal), and peak amplitude were obtained on each trial within the duration of the ISI after CS onset. Statistical testing of differences in means were conducted by 
independent and repeated-measures ANOVA, as well as independent and paired-sample $t$ tests.

Experimental design to detect latent learning in cerebellar cortex. The basic design of the three experiments was as follows. In phase I, experimental subjects were given pairings of a relatively long CS (750 or 800 $\mathrm{msec}$ ) and the US at an ISI of 700 or $750 \mathrm{msec}$. This training was either subthreshold (experiment 3 ) or was terminated at threshold levels before the emergence of robust conditioned responses (experiments 1 and 2). In phase II, subjects were given pairings of the same CS but of a relatively short duration ( 250 or $300 \mathrm{msec}$ ) and the US at an ISI of 200 or $250 \mathrm{msec}$. This training continued until robust conditioned responses were established (five or six sessions). Subjects were then retested with a relatively long CS, either of the original duration used in phase I (experiment 1) or an even longer duration (1000 or $1250 \mathrm{msec}$; experiments 2 and 3).

Experiment 1 tested the hypothesis that learning in the cerebellar cortex that was latent, because of absence of plasticity in the AIN, could later be expressed after establishing plasticity in the AIN by training with a different ISI (Fig. 1). To detect latent learning, we used the ISI-specific timing of learning in the cerebellar cortex and the ability to produce reversible disconnection of the cerebellar cortex to detect learning in the AIN. Each animal was initially tested for baseline responding with the cerebellar cortex disconnected via infusion of the GABA antagonist picrotoxin into the AIN (Fig. 1A1). Phase I training began the next day with daily training sessions using standard delay conditioning with a relatively long ISI. Four experimental subjects [group PP (paired)] were trained with a $700 \mathrm{msec}$ ISI and 11 others were trained with a $750 \mathrm{msec}$ ISI, and training continued until conditioned responses were observed on three of nine consecutive trials or until a fixed number of sessions (five and two, respectively) had elapsed (mean number of trials to criterion, 304 and 149, respectively). We hypothesized that, at this point, the cerebellar cortex had learned appropriately timed responses for the long ISI but that responses were small or absent because of the absence of learning in the AIN (Fig. $1 B$ ). To test the status of plasticity in the nucleus, each rabbit was tested the next day with an infusion of picrotoxin into the AIN (Fig. 1B1). Next, phase II training was designed to induce plasticity in the AIN. This entailed training subjects for five daily sessions to asymptote using a shorter ISI and CS duration (Fig. 1C). The four subjects trained with a $700 \mathrm{msec}$ ISI $(750 \mathrm{msec} C S)$ in phase I were trained with a $200 \mathrm{msec}$ ISI $(250 \mathrm{msec} C S)$, and 11 subjects trained with a $750 \mathrm{msec}$ ISI ( $800 \mathrm{msec} C S)$ in phase I were trained with a $250 \mathrm{msec}$ ISI (300 msec CS). A third picrotoxin infusion was conducted on the day after phase II training to test for learning in the AIN as indexed by the presence of short-latency responses (Fig. 1C1; see Criteria for cannula placements). The next day we tested for latent learning in phase I by presenting probe trials with the long CS. We hypothesized that these would elicit double-peaked responses. The first peak would reflect phase II training, whereas the second would reflect latent learning from phase I (Fig. 1D). Thus, the presence or absence of the second peaks in these probe trials constitutes the test for the induction of latent learning in phase I.

Experiment 2 tested whether the double-peaked responses observed were controlled by CS offset or by the ISI in phase I by testing subjects with probe trials longer than the CS used in phase I, and experiment 3 tested whether overt responding during phase I was a necessary condition for observing double-peaked responses by using a fixed number of phase I trials insufficient for establishing conditioned responses (see Results). For experiments 1 and 2, two control groups $(n=4$ and 8 each for experiments 1 and 2, respectively) were used to contrast with the experimental group PP to show that the double-peaked responses depend on the pairing of the tone and shock in phase I. These groups received the same treatment as group PP except for phase I. For subjects in group UP (unpaired), the tone and shock were given in an explicitly unpaired manner, in pseudo-Gellerman orders (Gellerman, 1933) and the number of tones and shocks presented to each animal was yoked with respect to subjects in group PP. Subjects in group NP (no training) were placed in the experimental chamber, with the time of exposure for each animal yoked to experimental subjects.

Criteria for cannula placements. To assess the effects of picrotoxin injections conducted at separate time points in experiment 1 , we established a set of criteria to determine whether the cannula placement was appropriate for a particular subject. The first criterion involved 12 and 4 subjects trained with a 200 and $250 \mathrm{msec}$ ISI, respectively, in phase II. These subjects were tested with six preinjection and six postinjection CS alone trials on the subsequent test day, and thus a one-tailed $t$ test was conducted between the criterion onset latencies of the last six condi-

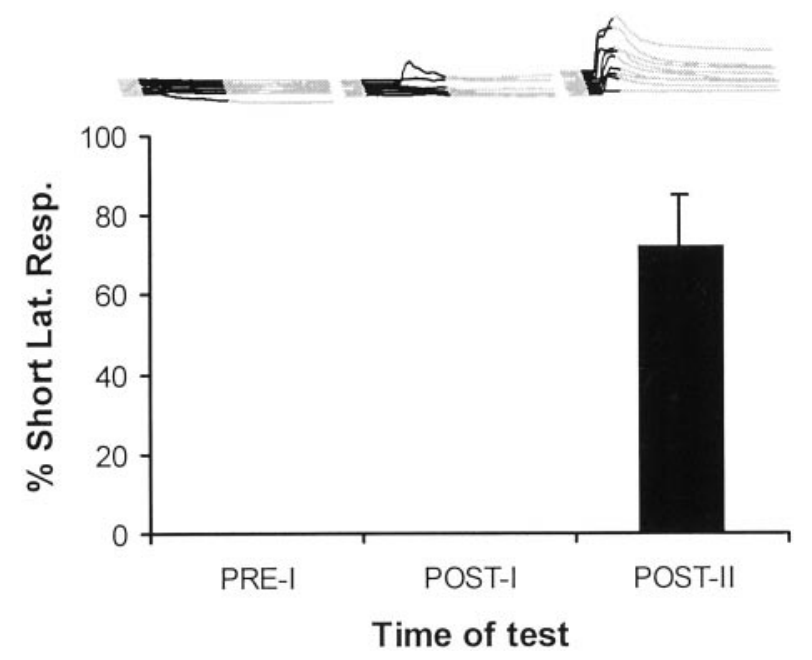

Figure 2. Percent trials with a short-latency response during the three test sessions with picrotoxin in experiment 1 . The data are collapsed across experimental subjects demonstrating a significant decrease in criterion onset latency during postinjection trials after phase II (for details, see Materials and Methods). Short-latency responses are absent before and after phase I (PRE-I, POST-I) but are present after phase II (POST$I I)$. The top panel from left to right shows individual response topographies on CS-alone trials presented under picrotoxin for a representative subject during tests conducted at each of the three separate time points.

tioned responses before injection and those of responses on the six postinjection trials. Cannula placement was considered appropriate if the postinjection latencies were significantly shorter than preinjection latencies $(p<0.01)$. Cannula placement was considered appropriate for one subject that did not meet this initial criterion, when criterion onset latencies of the first 12 conditioned responses after picrotoxin injection during a subsequent post-extinction drug test were seen to be significantly shorter than the last 12 conditioned responses before the first post-phase II test $(p<0.01)$. A second criterion involved seven experimental subjects trained with a $250 \mathrm{msec}$ ISI in phase II. These subjects were tested with picrotoxin during a full training session, and therefore a greater number of postinjection trials were available. The criterion onset latencies of the last 12 conditioned responses before picrotoxin injection were compared with those of the first 12 conditioned responses after injection, and cannula placement was considered appropriate if the postinjection latencies were significantly shorter than the preinjection latencies $(p<0.01)$. A short-latency response was defined as any conditioned response with a criterion onset latency shorter than the mean preinjection latency as defined by the above tests.

Analysis criteria for double-peaked responses. Responses on individual test trials with the long CS were classified as double-peaked responses for subjects exhibiting conditioned responses on more than one long CS test trial. For subjects trained with ISIs of 700 and $200 \mathrm{msec}$ in phases I and II, respectively, and tested with a 750 msec CS (experiment 1), peak latencies were obtained in two $400 \mathrm{msec}$ time windows each centered around the short and long ISI (0-400 and 500-900 msec). Responses were classified as double-peaked whenever the peak latency for the first window fell below $350 \mathrm{msec}$, that for the second window was at least 540 msec, and amplitudes of the first and second peaks were at least $0.3 \mathrm{~mm}$. For subjects trained with ISIs of 750 and $250 \mathrm{msec}$ in phases I and II, respectively, and tested with a $800 \mathrm{msec}$ CS (experiment 1 ) or with a 1000 or $1250 \mathrm{msec}$ CS (experiments 2 and 3), peak latencies were obtained in either two or three $500 \mathrm{msec}$ time windows each centered around the short and long ISIs (0-500 and 500-1000 msec; experiments 2 and 3 ) and CS offset (1000-1500 msec; experiment 3). Responses were classified as double-peaked when the latency of the first peak was less than $450 \mathrm{msec}$ and that of the second was at least $540 \mathrm{msec}$, and the amplitude of the second peak was at least $0.3 \mathrm{~mm}$. For experiments 2 and 3 , an additional requirement was that the latency of the second peak occur before the offset of the CS. Furthermore, in all cases, a response was classified as double-peaked only when the amplitude of the second peak was greater than the minimum between the first and second peaks by at least $1 \mathrm{~mm}$ or at least three times that value. 


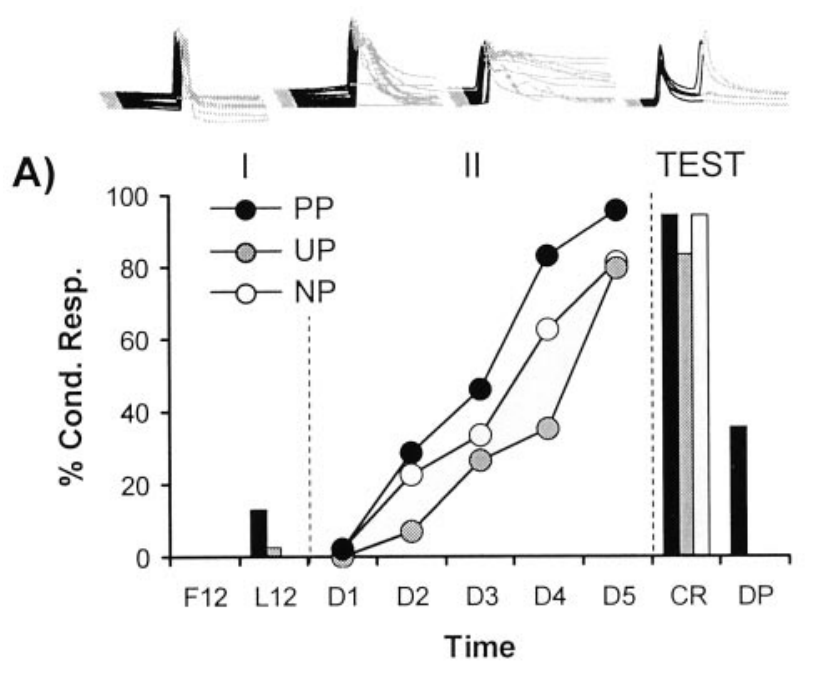

B)

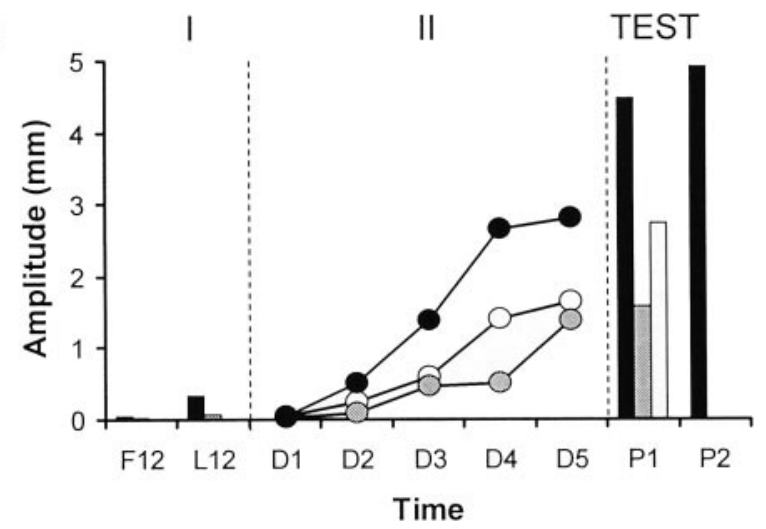

Figure 3. A, Percent trials with a conditioned response during the course of training and testing for groups PP, UP, and NP in experiment 1. F12 and $L 12$ refer, respectively, to the first and last 12 trials (both paired and CS-alone) of phase I, and D1-D5 indicate performance during the 12 CS-alone trials on each of the $5 \mathrm{~d}$ of phase II. Performance during the first six long CS trials is shown at the far right for subjects exhibiting more than one conditioned response during the test trials (TEST), in which $C R$ refers to all conditioned responses and $D P$ refers to conditioned responses with a second peak. Little conditioning was seen before or after phase I (left, I), but double-peaked responses were seen on long CS test trials presented after phase II only for experimental subjects (TEST). B, Conditioned response amplitude during the course of training and testing for groups PP, UP, and NP in experiment 1. Performance during the six long CS trials is shown at the far right for subjects exhibiting more than one conditioned response during the test trials (TEST), in which $P 1$ and $P 2$ refer, respectively, to the amplitudes of the first peak and second peaks. The amplitude of the second peak was obtained from only double-peaked trials (subjects exhibiting no such response were assigned a zero). The top panel shows response topographies for one subject in group PP at different stages of the experiment (first 12 trials of phase I, last 12 trials of phase I, last 12 trials of phase II, and first six long CS trials). Dark lines indicate the time at which the CS was present during the trial.

To assess whether the learning in phase I was latent in experiments 1 and 2, for each double-peaked responder, a paired-comparison $t$ test (one-tailed) was conducted between the maximum amplitude of a second peak observed across double-peaked responses and that of any response observed on the last 50 trials of phase I (for experiment 1, this included the preinjection trials of the post-phase I test). To the extent that learning in phase I was latent, we expected the maximum amplitude of second peaks to be significantly higher than that of any response observed to the long CS near the end of phase I (for discussions on criteria for establishing latent learning, see Thistlethwaite, 1951).

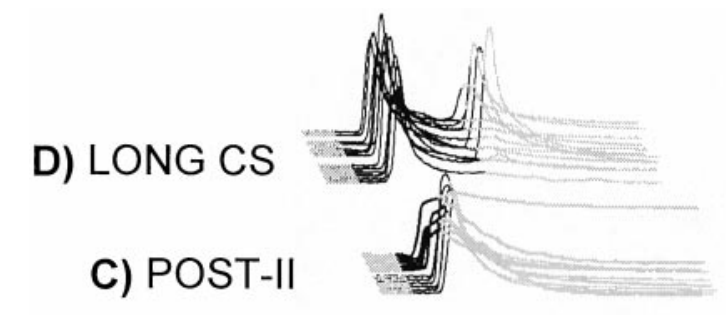

\section{B) POST-I}

\section{A) PRE-I}

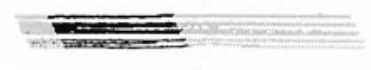

Figure 4. Individual response topographies during the three picrotoxin tests and test trials with the long CS for a representative subject in group PP. Dark lines indicate the time during which the tone CS was present during the trial. Bold lines indicate trials during which picrotoxin was in effect.

\section{RESULTS}

\section{Experiment 1: nucleus plasticity}

We hypothesized that plasticity in the AIN, as assessed by the presence of short-latency responses to the CS under the influence of picrotoxin, would be absent or too small to measure before and after phase I and robust after conditioning with the short ISI in phase II (Fig. 1A1-C1). Of the four (group PP) and 11 experimental subjects (the latter trained with longer ISIs), one and five subjects, respectively, exhibited responses with shortened onset latencies under picrotoxin after phase II, satisfying the criteria for appropriate cannula placement (for details, see Materials and Methods). For these six animals, no short-latency responses were observed before or after phase I training, whereas robust levels were observed after phase II (Fig. 2). This is consistent with the previous finding that acquisition of short-latency responses parallels that of timed conditioned responses (J. F. Medina, K. S. Garcia, and M. D. Mauk, unpublished observations). For all experimental subjects, phase I training produced little or no increase in percent trials with a conditioned response and response amplitude, whereas phase II training resulted in robust conditioning (Fig. 3; data shown for group PP). This pattern of changes did not differ from subjects given explicitly unpaired training with number of stimuli yoked to experimental subjects (group UP; $n=4$ ), and the increase in phase II did not differ from subjects given yoked exposure to the experimental chamber in phase I (group NP; $n=4$ ) (Fig. 3). Mean percent trials with a short-latency response on the first six trials under the influence of picrotoxin on the three test days (pre-phase I, post-phase I, and post-phase II) were 0,0 , and $72.2 \%$, respectively, and a one-way ANOVA revealed a highly significant effect of time of test $\left(F_{(2,12)}\right.$ $=32.5 ; p<0.001)$. The percent short-latency responses during the post-phase II test were significantly higher compared with either pre-phase I or post-phase I tests $(t$ values $>6.65$; $p$ values $<$ $0.005)$. Mean percent trials with a conditioned response for groups PP and UP on the first and last 12 trials of phase I were 0 and 12.9 , and 0 and $2.8 \%$, and corresponding response amplitudes were 0.04 and 0.33 , and 0.03 and $0.06 \mathrm{~mm}$. Independent two-way ANOVAs revealed an effect of test time for percent trials $\left(F_{(1,12)}\right.$ $=6.48 ; p<0.05)$ but not for response amplitude $\left(F_{(1,12)}=3.71\right)$. The mean percent CS-alone trials with a conditioned response increased for all groups during phase II.A two-way ANOVA 
revealed a highly significant effect of day $\left(F_{(4,45)}=16.0 ; p<0.001\right)$ but neither an effect of group $\left(F_{(2,45)}=2.73\right)$ nor day $\times$ group interaction $\left(F_{(8,45)}<1\right)$. The mean response amplitude also increased for all groups. A two-way ANOVA revealed a significant effect of day $\left(F_{(4,45)}=7.63 ; p<0.001\right)$ as well as an effect of group $\left(F_{(2,45)}=5.03 ; p<0.05\right)$ but no day $\times$ group interaction $\left(F_{(8,45)}<1\right)$. Equivalent results were obtained for the 11 experimental subjects trained with longer ISIs. Mean percent trials with a conditioned response on the first and last 12 trials of phase I were 0.8 and $7.6 \%$, and corresponding response amplitudes were 0.03 and $0.12 \mathrm{~mm}$. Although both measures increased ( $t$ values $>$ 2.34 ; $p$ values $<0.05)$, response amplitudes remained substantially below the criterion threshold. Both mean percent CS-alone trials with a conditioned response and response amplitude increased during phase II. One-way ANOVAs revealed highly significant effects of day for percent trials $\left(F_{(4,40)}=36.4 ; p<0.001\right)$ and response amplitude $\left(F_{(4,40)}=38.7 ; p<0.001\right)$.

\section{Experiment 1: latent learning}

During the crucial test with the long CS, only experimental subjects exhibited double-peaked responses timed to the long ISI used in phase I, consistent with the hypothesis that latent learning occurs in the cerebellar cortex. None of the control subjects exhibited double-peaked responses, suggesting that the second peak was associative and not attributable to other factors, such as stimulus generalization from short to long tones (Fig. 3A, right). Individual responses given during the three picrotoxin tests and the final test with the long CS are shown for a representative subject in group PP (Fig. 4).

Mean percent long CS trials (first six) with a conditioned response were 94.4, 83.3, and 94.4\%, respectively, and an independent one-way ANOVA revealed no effect of group $\left(F_{(2,6)}<1\right.$; $p=0.49$ ). In contrast, mean percent long CS trials (first six) with double-peaked responses for groups PP, UP, and NP were 35.6, 0, and $0 \%$, respectively. An independent one-way ANOVA revealed a significant effect of group $\left(F_{(2,6)}=12.96 ; p<0.01\right)$, and a $\chi^{2}$ test on frequencies of subjects exhibiting single-peaked or doublepeaked responses in each group revealed a significant departure from independence $\left(\chi_{(2)}^{2}=9.0 ; p<0.05\right)$. Mean peak latencies in the first time window for trials with a conditioned response in groups PP, UP, and NP were 235, 244, and $239 \mathrm{msec}$, respectively, and an independent one-way ANOVA revealed no effect of group $\left(F_{(2,6)}<1\right)$. The mean peak latency in the second window for trials with a double-peaked response in group PP was $681 \mathrm{msec}$. For the 11 other experimental subjects, six exhibited at least one double-peaked response on long CS trials (six presented across $2 \mathrm{~d}$ of extinction or during an initial day of extinction for four and seven subjects, respectively). The mean percent trials with double-peaked responses across subjects was 9.4\%. (The low level of double-peaked responses compared with group PP most likely reflects the effect of distributing the test trials among short CS extinction trials.) The mean peak latency in the first time window for trials with a conditioned response was $303 \mathrm{msec}$, and that in the second window on trials with a double-peaked response across subjects exhibiting at least one double-peaked response was 833 msec.

For the nine experimental subjects exhibiting a double-peaked response, the amplitude of the second peak was significantly greater than that of any response before phase II $\left(t_{(8)}=3.45 ; p<\right.$ $0.01)$, consistent with the view that learning in phase I was latent.
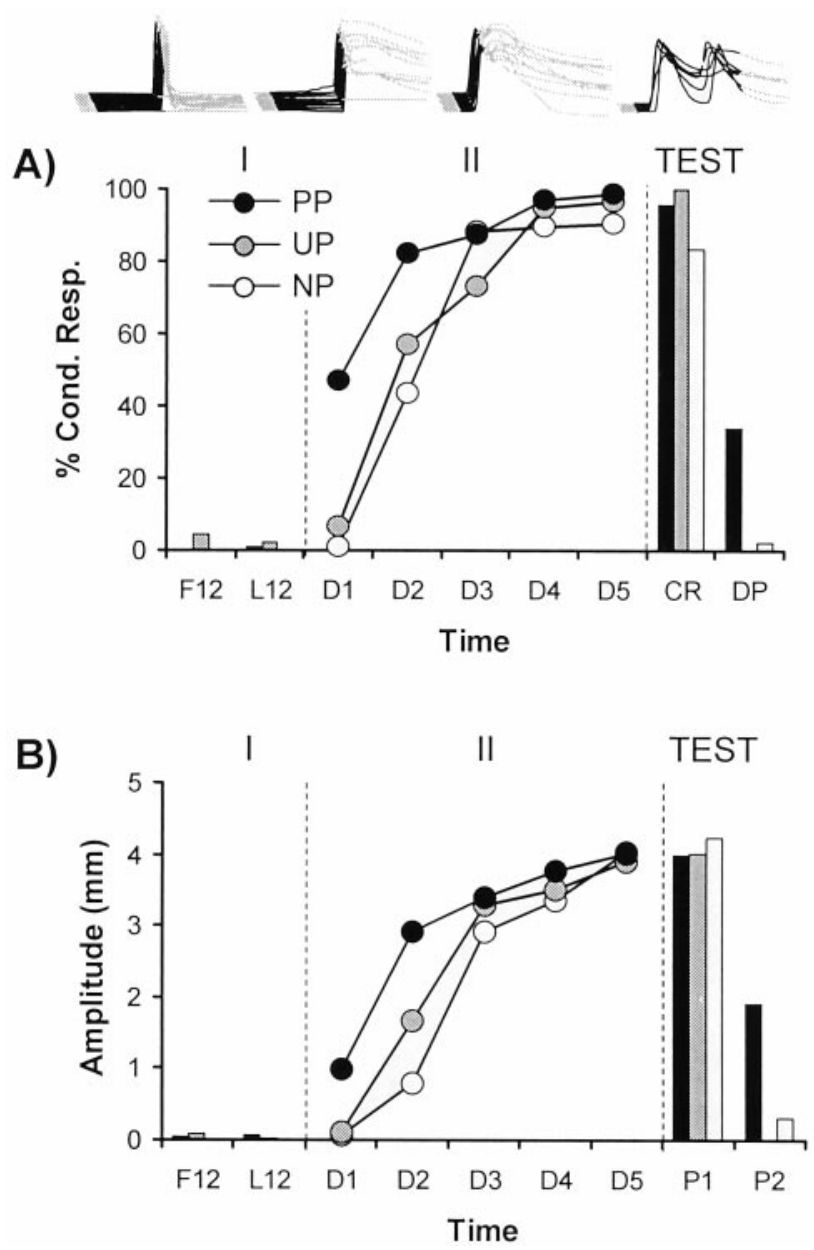

Figure 5. A, Percent trials with a conditioned response during the course of training and testing for groups PP, UP, and NP in experiment 2. Labels are marked as in Figure 2. Double-peaked responses were observed only in group PP (TEST). B, Conditioned response amplitude during the course of training and testing for groups PP, UP, and NP in experiment 2. The top panel shows response topographies for one subject in group PP at different stages of the experiment (first 12 trials of phase I, last 12 trials of phase I, last 12 trials of phase II, and first six long CS trials) tested with a $1250 \mathrm{msec}$ CS. Dark lines indicate the time at which the CS was present during the trial.

\section{Experiment 2: discriminating latent learning from CS-offset responses}

In experiment 1 , the second peak latency on long CS trials showed a tendency to be near the time of CS termination (Fig. 4). Experiment 2 examined the possibility that the second peaks on long CS test trials occur in response to the termination of the CS rather than on the basis of latent learning established in phase I. We trained eight rabbits (group PP) without cannula under similar conditioning parameters and subsequently tested them with a CS longer than that used in phase I. Phase I training (approximately four sessions) with a $750 \mathrm{msec}$ ISI (800 msec CS) continued until the same criterion level was met as in the previous experiments (mean number trials to criterion, 211). During the next $5 \mathrm{~d}$, subjects were given training with a $250 \mathrm{msec}$ ISI. A second group UP $(n=8)$ received explicitly unpaired trials, and a third group NP $(n=8)$ received no training in phase I. After phase II, subjects were tested with $18 \mathrm{CS}$-alone trials that were either 1000 ( $n=4$ per group) or 1250 ( $n=4$ per group) msec in duration. If the second peak reflects timing of the ISI in phase I 
$1000 \mathrm{~ms}$

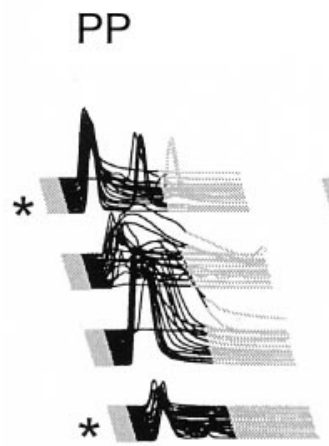

UP

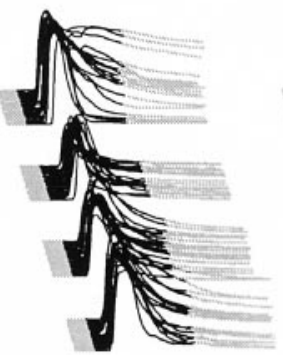

NP

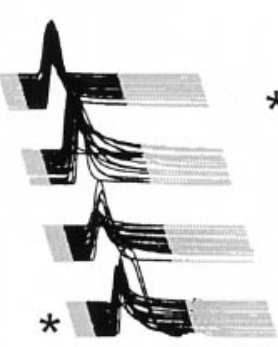

PP

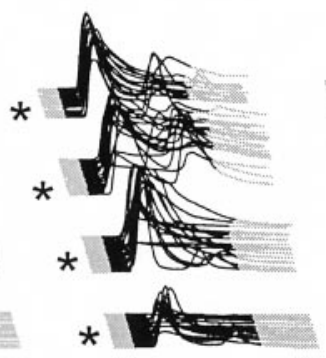

$1250 \mathrm{~ms}$

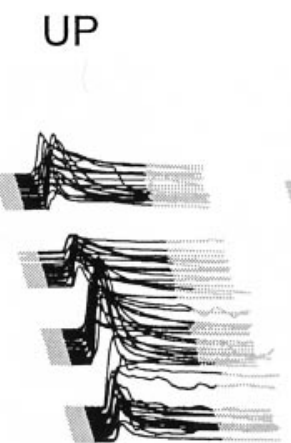

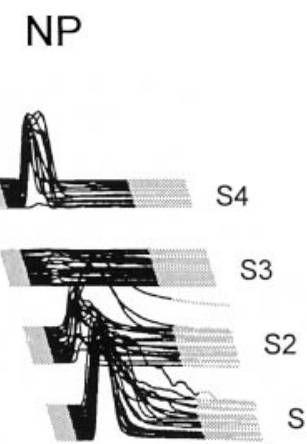

Figure 6. Response topographies during 18 long CS test trials for all subjects in experiment 2, tested with either a 1000 or 1250 msec tone. Dark lines indicate the time at which the tone CS was present during the trial. Asterisks indicate subjects exhibiting at least one double-peaked response during the first six test trials. Double-peaked responses were observed in group PP but not in groups UP or NP. The second peaks occurred before the termination of the CS, near the ISI used in phase I.

rather than in response to CS offset, then its peak latency should occur before the termination of long CS trials.

As in experiment 1 , the mean percent trials with a conditioned response and response amplitude exhibited little change after phase I but increased substantially during phase II training (Fig. 5). The rate of conditioned response acquisition, as measured by the change in percent trials with a conditioned response, was slightly higher in group PP than in groups UP or NP, suggesting that there was some savings in learning from phase I to phase II.

The mean percent trials with a conditioned response for groups PP and UP on the first and last 12 trials of phase I were 0 and 1.0 , and 4.5 and $2.2 \%$, whereas corresponding mean response amplitudes were, 0.05 and 0.06 , and 0.08 and 0.02 $\mathrm{mm}$. Independent two-way ANOVAs revealed neither an effect of time of test for percent trials $\left(F_{(1,28)}<1\right.$; $\left.p=0.68\right)$ nor response amplitude $\left(F_{(1,28)}=4.04 ; p=0.054\right)$, indicating that there was no significant increase in either measure during phase I. The mean percent CS-alone trials with a conditioned response increased for all groups during phase II. A two-way ANOVA revealed highly significant effects of day $\left(F_{(4,105)}=\right.$ $50.29 ; p<0.001)$, group $\left(F_{(2,105)}=9.27 ; p<0.001\right)$, and day $\times$ group interaction $\left(F_{(8,105)}=2.22 ; p<0.05\right)$. Independent oneway ANOVAs at each day revealed a significant effect of group only on the first day of phase II $\left(F_{(2,21)}=11.93 ; p<0.001\right)$, and the differences between groups PP and UP and between groups PP and NP were both significant ( $t$ values $>3.56$; $p$ values $<0.01$ ). Similarly, mean response amplitude increased for all groups during phase II, and a two-way ANOVA revealed a highly significant effect of day $\left(F_{(4,105)}=33.56 ; p<0.001\right)$ and a significant effect of group $\left(F_{(2,105)}=4.11 ; p<0.05\right)$. There was no day $\times$ group interaction $\left(F_{(8,105)}<1 ; p=0.49\right)$, indicating that the increase in amplitude did not differ among groups.

During the crucial test with the long CS, subjects in group PP exhibited double-peaked responses that occurred before the termination of the CS and timed near the ISI in phase I (Figs. $5 A$, right, $6, P P)$. Control subjects showed little or no evidence of a second peak (Figs. 5A, right, 6, UP, NP). These results confirm that the second peak was timed to the ISI used in phase I and was not controlled by the termination of the CS (but see Discussion).

The mean percent long CS trials with a conditioned response (first six) for groups PP, UP, and NP were 95.8, 100, and 83.3\%, respectively, and an independent one-way ANOVA revealed no effect of group $\left(F_{(2,21)}=1.44 ; p=0.26\right)$. In contrast, 6,0 , and 1 subjects, respectively, in groups PP, UP, and NP exhibited double-peaked responses (for definition, see Methods and Materials) on these trials. The mean percent trials with a doublepeaked response were $33.8,0$, and $2.1 \%$, respectively, for groups PP, UP, and NP. An independent one-way ANOVA revealed a significant effect of group $\left(F_{(2,21)}=9.61 ; p<0.01\right)$, and paired comparisons revealed that the percent trials for group PP was greater than for either group UP or NP ( $t$ values $>3.64 ; p$ values $<0.01$ ), whereas those for groups UP and NP did not differ $\left(t_{(7)}=1.51 ; p=0.18\right)$. A $\chi^{2}$ test on the frequencies of subjects exhibiting double-peakedresponses revealed a significant departure from independence $\left(\chi_{(2)}^{2}=11.87 ; p<0.005\right)$. Mean latencies to the first peak on trials with a conditioned response were 287, 284, and $266 \mathrm{msec}$, respectively, for groups PP, UP, and NP, and a one-way ANOVA revealed no effect of group $\left(F_{(2,20)}=\right.$ $1.86 ; p=0.18)$. The mean latency to the second peak on trials with a double-peaked response across the six subjects of group PP was 845 msec.

As in experiment 1 , for the six experimental subjects exhibiting a double-peaked response, the amplitude of the second peak was significantly greater than that of any response before phase II $\left(t_{(5)}=4.15 ; p<0.01\right)$, again consistent with the view that learning in phase I was latent.

\section{Experiment 3: necessity of overt conditioning in phase I}

It is clear from the above experiments that phase II training with the short ISI produces a significant enhancement in the timed response to the long ISI used in phase I, observed in the form of a second peak on long CS test trials. Nevertheless, in both experiments, phase I training generally continued until overt responses could be observed, albeit at a low level and with small amplitudes. It is therefore possible that responding is still necessary for the second peak to be manifest on long CS test trials and that, in this sense, the learning in phase I is not truly latent. To address this question, we trained a group of subjects in a final experiment with a fixed number of phase I trials that we knew would be insufficient to produce overt conditioning. Eight subjects were given $30 \mathrm{CS}-\mathrm{US}$ pairings in phase I at a $750 \mathrm{msec}$ ISI, 

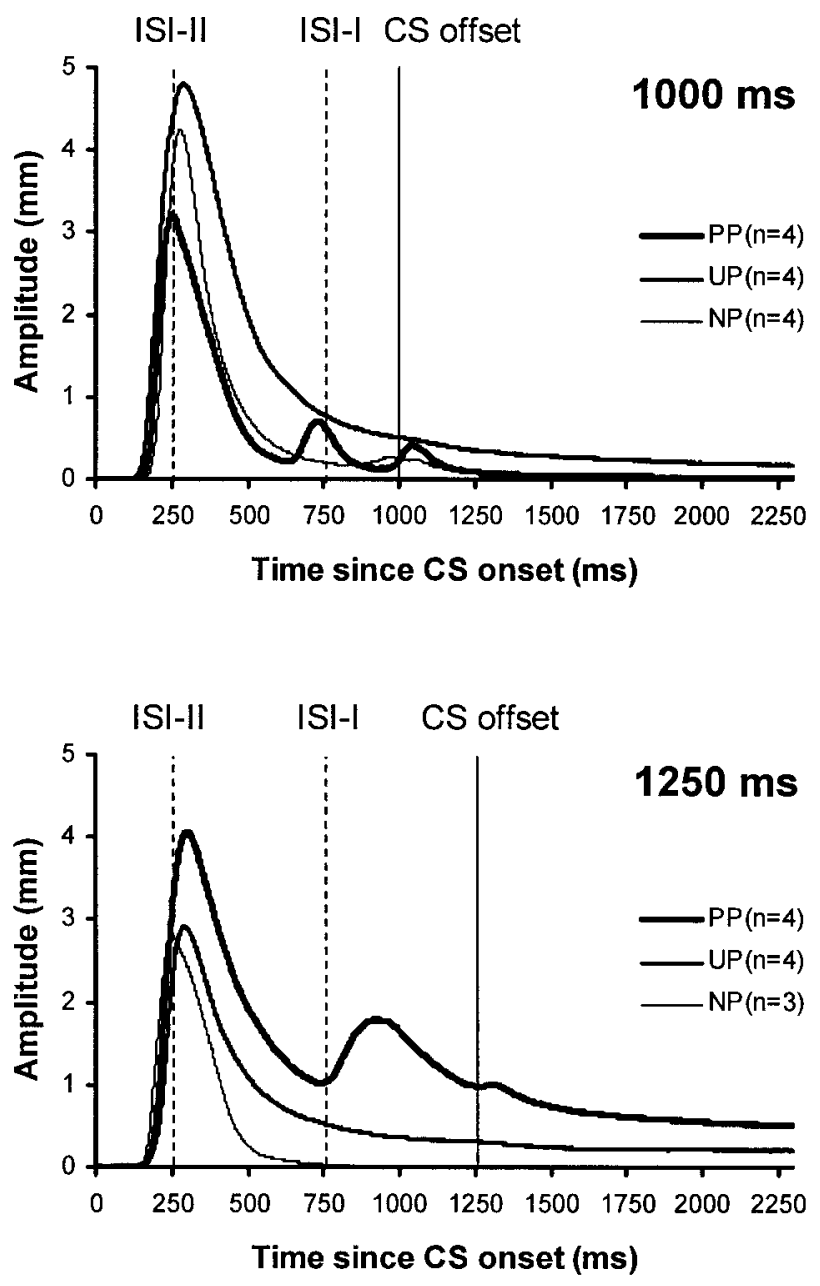

Figure 7. Response topographies averaged across the first six long CS test trials in experiment 2, for subgroups of subjects tested with a 1000 or $1250 \mathrm{msec}$ CS. Peaks were observed near the ISI used in phase I, as well as near the end of the test CS. The duration of the test CS appears to influence the latency of the second peak. Dotted vertical lines indicate the ISIs in phases I and II, and solid vertical lines indicate the time of CS offset.

followed by six sessions of phase II training at a $250 \mathrm{msec}$ ISI (96 paired and $12 \mathrm{CS}$-alone trials per session; one extra session was given to ensure robust responding appropriate for the short ISI), and subsequently tested with 18 long CS trials $(1250 \mathrm{msec})$. As above, the test of the latent learning hypothesis involves the presence or absence of double-peaked responses.

Although none of the subjects exhibited any conditioned response in phase I, six subjects exhibited double-peaked responses during the crucial test with the long CS. The percent long CS trials (first six) with a double-peaked response (with second peak latencies occurring before $1250 \mathrm{msec}$ ) was $18.8 \%$ across the eight subjects. A one-way ANOVA conducted on the percent trials in this group and in control subjects in experiment 2 tested with a 1250 msec CS ( $n=4$ each in groups UP and NP) revealed a significant effect of group $\left(F_{(1,14)}=10.31 ; p<0.01\right)$. Furthermore, a $\chi^{2}$ test on the frequencies of double-peaked responders (excluding one subject that failed to exhibit conditioned responses in group NP) revealed a significant departure from independence $\left(\chi_{(1)}^{2}=8.75 ; p<0.005\right)$. The results thus show that responding in phase $\mathrm{I}$ is not required for observing double-peaked responses after phase II and provide strong evidence for the latent learning hypothesis.

\section{DISCUSSION}

The present results show that latent learning that is temporally specific to the ISI is established by subthreshold or threshold-level training with a long ISI. This learning was expressed in the form of double-peaked responses to long CS probe trials presented after robust conditioning with a short ISI. Both experiments 1 and 2 showed that the double-peaked responses were associative in nature and that the second peak was slightly delayed but generally appropriately timed for the ISI in phase I. Experiment 1 showed that the emergence of plasticity in the AIN was correlated with the emergence of conditioned responding. Short-latency responses were observed neither before nor after phase I training but after robust conditioning with the short ISI. Experiment 2 showed that the second peak was not a response to the termination of the CS but reflected latent learning associated with the ISI in phase I. Experiment 3 showed that double-peaked responses could be observed with an amount of phase I training insufficient to produce overt conditioned responding.

Previous studies demonstrating double-peaked responses under mixed ISI training (Hoehler and Leonard, 1976; Millenson et al., 1977) provided evidence for a learned timing mechanism. The present results show that this mechanism is engaged even before the expression of robust conditioned responding. Our data are consistent with the notion that there are at least two sites of plasticity in the cerebellum, one involved in the timing of the conditioned response and another that is permissive for its expression (cf. Mauk and Donegan, 1997). The data are consistent with the hypothesis that, even without robust conditioned response expression, learning temporally specific to the ISI is initially established in the ipsilateral cerebellar cortex. Reversible disconnection of the cerebellar cortex shows that this learning occurs before detectable plasticity is established in the AIN. Subsequent conditioning with a shorter ISI in phase II induced plasticity in the AIN, as suggested by the presence of shortlatency responses. That this additional plasticity allows plasticity in the cerebellar cortex to be expressed as a double-peaked response on long CS trials is consistent with the view that the former plays a permissive role in conditioned response expression. That the timing of the second peak was appropriate for the long ISI is consistent with the hypothesis that plasticity in the cerebellar cortex is responsible for conditioned response timing.

The experimental subjects in experiments 2 and 3, when tested with a CS duration longer than originally used in phase I, exhibited peaks timed to occur near the offset of the CS with repeated test trials. Such responses were observed in the form of an occasional small third peak or a second peak occurring near CS offset during a third time window (1000-1500 msec from CS onset) and are reflected in the average response topographies for subjects in experiment 2 (Fig. 7). All six subjects exhibiting double-peaked responses showed a trend in which the latency of the second peak shifted toward the time of CS offset with repeated test trials (Fig. 8). These results suggest that there is a contribution of the CS offset to the double-peaked responses observed in experiment 1 . It is important to note that the responses occur not in response to, but rather, in anticipation of, the termination of the CS. In addition to the ISI in phase I, experimental subjects appear to learn that the US occurs near the termination of the CS and use this information to adjust their responses to coincide with $\mathrm{CS}$ offset. That such responses rarely 

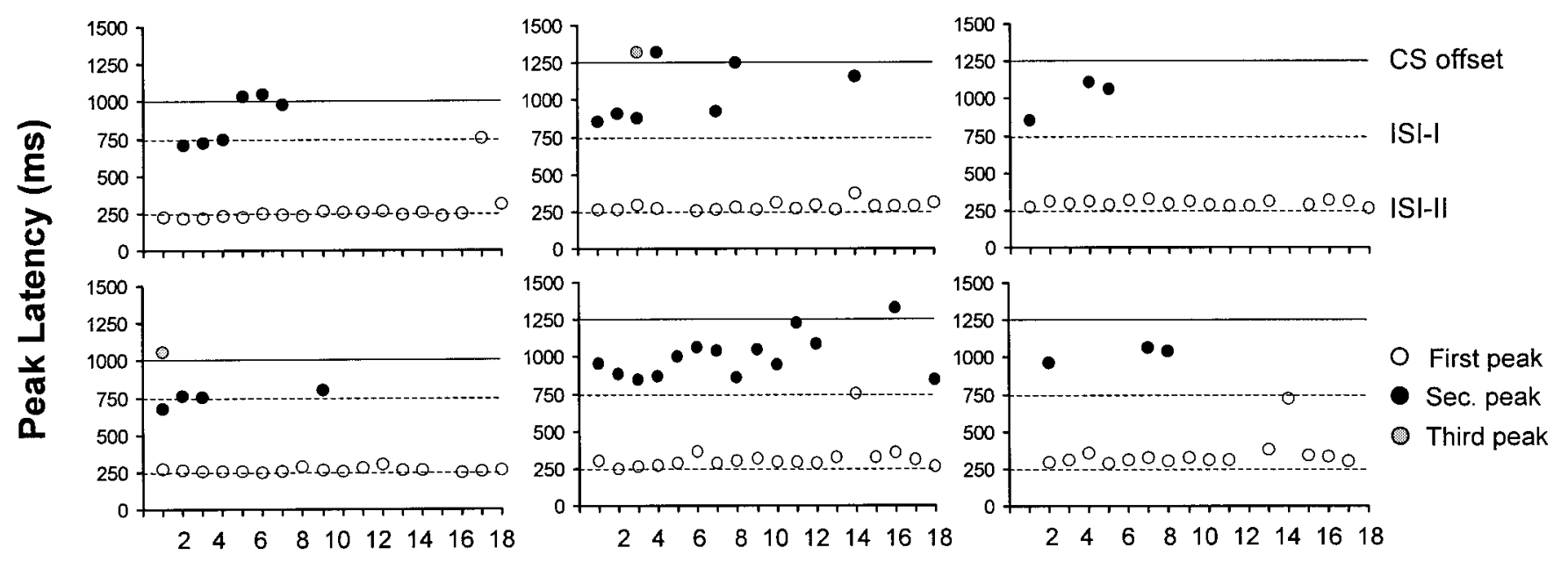

\section{Trial}

Figure 8. Peak latencies of first and second peaks on double-peaked trials plotted across the 18 test trials for individual subjects of group PP in experiment 2. White, black, and gray circles represent first, second, and third peaks, respectively. On occasional trials, a triple-peaked response is observed, with each peak timed, respectively, to the ISIs in phases II and I as well as CS offset. Whereas the peak latency of the first peak remains unchanged during testing, that of the second peak increased across test trials. Dotted lines indicate the ISIs in phases I and II, and solid lines indicate the time of CS offset.

occurred in control subjects suggests that they are nevertheless associative in nature and require experience with at least two ISIs. The mechanisms underlying such learning are unclear. Extracerebellar mechanisms may be responsible for this rapid adjustment in response timing. Alternatively, repeated testing with a long CS in extinction may increase the latency of the second peak, via incidental pairings of granule cell activity persisting during the extended CS with rebound excitation in climbing fiber activity upon US omission. Further tests are required to distinguish between these alternative interpretations.

What is the amount of phase I training required for the doublepeaked responses to first appear? Experiment 3 showed that double-peaked responses could be observed with as few as 30 phase I trials, an amount of training that produced no overt conditioned responding. This result is consistent with the computer simulations of Medina and Mauk (1999), which showed that the plasticity rules producing stable learning were those that induced initial plasticity in the cerebellar cortex and subsequent plasticity in the AIN. A study by Ross and Scavio (1983) using similar parameters suggests that double-peaked responses may be observed with as few as 15 training trials in phase I. They gave 15 pairings of a tone CS and shock US to separate groups of rabbits at different ISIs ranging from 0 to $4000 \mathrm{msec}$ and then conducted a transfer test in which all subjects were given 320 CS-US pairings with a $500 \mathrm{msec}$ ISI. They found that learning was facilitated for nearly all ISIs in phase I (except for 0 and 200 $\mathrm{msec}$ ), in particular for those near $500 \mathrm{msec}$. To the extent that this facilitation is mediated by the same mechanisms that are involved in the latent learning observed in our experiments, double-peaked responses may be expected with 15 phase I trials, an amount of training substantially less than that normally required for overt conditioning. The rate of learning in the cerebellar cortex might be determined independently of learning in the AIN, at least under the current training parameters, by varying the number of trials in phase I from 1 to 15 trials.

Our findings also raise questions regarding the conclusions of a related study in which learning with a long ISI during reversible inactivation of the AIN was later expressed as a double-peaked response to the long CS after robust conditioning with a short ISI (Yeo et al., 1997). In this study, the authors infused muscimol, a GABA-A agonist that hyperpolarizes target cells, into the AIN to prevent the learning and expression of conditioned responses. The authors reported that their infusions of muscimol into the AIN were not restricted to the cerebellar nuclei, and therefore, produced a general inactivation of the ipsilateral cerebellum (Yeo et al., 1997). Because there was a complete absence of responses on CS-alone trials during muscimol inactivation, and yet subsequent conditioning with a short ISI revealed learning in phase I, the authors suggested that whatever changes that occurred during phase I must have been mediated by circuitry outside of the ipsilateral cerebellum (e.g., the contralateral cerebellum). Our results also show that, even in the virtual absence of conditioned responses, training with the long ISI produces learning and suggest that this learning may be mediated by the cerebellar cortex. Although Yeo et al. (1997) argued that their muscimol infusions probably disrupted normal functioning of the ipsilateral cerebellum by suppressing inhibitory feedback from the AIN to the inferior olive, this does not preclude the possibility that latent learning might have been established in the cerebellar cortex. Furthermore, their study did not distinguish between second peaks controlled by the ISI in phase I from those controlled by anticipation of CS offset. Our findings suggests that a significant portion of the second peaks observed in both experiments may require the ipsilateral cerebellar cortex. If this is the case, doublepeaked responses should be seen even if the contralateral cerebellum is removed. If the contralateral cerebellum is involved, as Yeo et al. (1997) suggest, then the second peak should be abolished.

Finally, latent learning has had an important status in traditional learning theory. Early studies (cf. Blodgett, 1929; Tolman and Honzik, 1930) showed clearly that learning could occur in the absence of performance, compelling psychologists to recognize the distinction between learning and performance. However, the mechanisms underlying latent learning have never been com- 
pletely specified, and few learning theories since have addressed the problem (but see Kehoe, 1988, 1992). A priori predictions of latent learning based on the core hypotheses of a biologically inspired model of conditioning were confirmed in the present study. Our results extend the range of latent learning phenomena to the temporal domain, because previous studies have been restricted in large part to the spatial domain (cf. Thistlethwaite, 1951). Alternative computational models of eyelid conditioning and timing based on hypothetical trace processes and neuron-like elements may provide ad hoc explanations of the present results (Desmond and Moore, 1988; Kehoe, 1988; Grossberg and Schmajuk, 1989; Wagner and Brandon, 1989; Bartha et al., 1991; Moore, 1992; Bullock et al., 1994; Fiala et al., 1996; Moore and Choi, 1997). Nevertheless, our study demonstrates the predictive power of hypotheses that are strongly constrained by the structure and physiology of the cerebellum (cf. Marr, 1969; Albus, 1971; Ito, 1984).

\section{REFERENCES}

Aizenman CD, Linden DJ (2000) Rapid synaptically driven increases in the intrinsic excitability of cerebellar deep nuclear neurons. Nat Neurosci 3:109-111.

Albus JS (1971) A theory of cerebellar function. Math Biosci 10:25-61.

Bartha GT, Thompson RF, Gluck MA (1991) Sensorimotor learning and the cerebellum. In: Visual structure and integrated functions (Arbib M, Ewert J, eds), pp 381-396. New York: Springer.

Blodgett HC (1929) The effect of the introduction of reward upon the maze performance of rats. Univ Calif Publ Psychol 4:113-134.

Bullock D, Fiala JC, Grossberg S (1994) A neural model of timed response learning in the cerebellum. Neural Networks 7:1101-1114.

Clark GA, McCormick DA, Lavond DG, Thompson RF (1984) Effects of lesions of cerebellar nuclei on conditioned behavioral and hippocampal neuronal responses. Brain Res 291:125-136.

Clark RE, Zhang AA, Lavond DG (1992) Reversible lesions of the cerebellar interpositus nucleus during acquisition and retention of a classically conditioned behavior. Behav Neurosci 106:879-888.

Desmond JE, Moore JW (1988) Adaptive timing in neural networks: the conditioned response. Biol Cybern 58:405-415.

Fiala JC, Grossberg S, Bullock DJ (1996) Metabotropic glutamate receptor activation in cerebellar Purkinje cells as substrate for adaptive timing of the classically conditioned eye-blink response. J Neurosci $16: 3760-3774$.

Garcia KS, Mauk MD (1998) Pharmacological analysis of cerebellar contributions to the timing and expression of conditioned eyelid responses. Neuropharmacology 37:471-480.

Garcia KS, Steele PM, Mauk MD (1999) Cerebellar cortex lesions prevent acquisition of conditioned eyelid responses. J Neurosci 19:10940-10947.

Gellerman LW (1933) Chance orders of alternating stimuli in visual discrimination experiments. J Genet Psychol 42:206-208.

Grossberg S, Schmajuk NA (1989) Neural dynamics of adaptive timing and temporal discrimination during associative learning. Neural Networks 2:79-102.

Hoehler FK, Leonard DW (1976) Double responding in classical nictitating membrane conditioning with a single-CS, dual-ISI pairing. Pavlov J Biol Sci 11:180-190.
Ito M (1984) The cerebellum and neural control. New York: Raven.

Kehoe EJ (1988) A layered network model of associative learning: learning to learn and configuration. Psychol Rev 95:411-433.

Kehoe EJ (1992) Versatility in conditioning: a layered network model. In: Motivation, emotion, and goal direction in neural networks (Levine DS, Leven SJ, eds), pp 90. Hillsdale, NJ: Erlbaum.

Krupa DJ, Thompson RF (1997) Reversible inactivation of the cerebellar interpositus nucleus completely prevents acquisition of the classically conditioned eye-blink response. Learn Mem 3:545-556.

Marr D (1969) A theory of cerebellar cortex. J Physiol (Lond) 202:437-470.

Mauk MD (1997) Roles of cerebellar cortex and nuclei in motor learning: contradictions or clues? Neuron 18:343-346.

Mauk MD, Donegan NH (1997) A model of Pavlovian eyelid conditioning based on the synaptic organization of the cerebellum. Learn Mem 3:130-158.

McCormick DA, Thompson RF (1984) Cerebellum: essential involvement in the classically conditioned eyelid response. Science 223:296-299.

Medina J, Mauk MD (1999) Simulations of cerebellar motor learning: computational analysis of plasticity at the mossy fiber to deep nucleus synapse. J Neurosci 19:7140-7151.

Medina JF, Garcia KS, Nores WL, Taylor NM, Mauk MD (2000) Timing mechanisms in the cerebellum: testing predictions of a large-scale computer simulation. J Neurosci 20:5516-5525.

Millenson JR, Kehoe EJ, Gormezano I (1977) Classical conditioning of the rabbit's nictitating membrane response under fixed and mixed CS-US intervals. Learn Motiv 8:351-366.

Moore JW (1992) A mechanism for timing conditioned responses. In: Time, action, and cognition (Macar E, ed), pp 229-238. Dordrecht: The Netherlands: Kluwer.

Moore JW, Choi JS (1997) The TD model of classical conditioning: Response topography and brain implementation. In: Neural-network models of cognition, Biobehavioral foundations, Advances in psychology, Vol 121 (Donahoe JW, Dorsel VP, eds), pp 387-405. Amsterdam, The Netherlands: North-Holland/Elsevier Science.

Perrett SP, Mauk MD (1995) Extinction of conditioned eyelid responses requires the anterior lobe of cerebellar cortex. J Neurosci 15:2074-2080.

Perrett SP, Ruiz BP, Mauk MD (1993) Cerebellar cortex lesions disrupt the learning-dependent timing of conditioned eyelid responses J Neurosci 13:1708-1718.

Raymond JL, Lisberger SG, Mauk MD (1996) The cerebellum: a neuronal learning machine? Science 272:1126-1131.

Ross RT, Scavio Jr MJ (1983) Perseveration of associative strength in rabbit nictitating membrane conditioning following ISI shifts. Anim Learn Behav 11:435-438.

Thistlethwaite D (1951) A critical review of latent learning and related experiments. Psychol Bull 48:97-129.

Tolman EC, Honzik CH (1930) Introduction and removal of reward, and maze performance in rats. Univ Calif Publ Psychol 4:257-275.

Wagner AR, Brandon SE (1989) Evolution of a structured connectionist model of Pavlovian conditioning (AESOP) In: Contemporary learning theories: Pavlovian conditioning and the status of traditional learning theory (Klein SB, Mowrer RR, eds), pp 149-189. Hillsdale, NJ: Erlbaum.

Yeo CH, Hardiman MJ, Glickstein M (1985) Classical conditioning of the nictitating membrane response of the rabbit. I. Lesions of the cerebellar nuclei. Exp Brain Res 60:87-98.

Yeo CH, Lobo DH, Baum A (1997) Acquisition of a new-latency conditioned nictitating membrane response-major, but not complete, dependence on the ipsilateral cerebellum. Learn Mem 3:557-577. 